University of Nebraska - Lincoln

DigitalCommons@University of Nebraska - Lincoln

May 2003

\title{
Ferromagnetic domain-wall behavior during reversal and thermally activated antiferromagnetic reversal in an exchange- biased NiO/NiFe bilayer
}

\section{Z. Y. Liu}

University of Nebraska - Lincoln

Shireen Adenwalla

University of Nebraska-Lincoln, sadenwalla1@unl.edu

Follow this and additional works at: https://digitalcommons.unl.edu/mrsecfacpubs

Part of the Materials Science and Engineering Commons

Liu, Z. Y. and Adenwalla, Shireen, "Ferromagnetic domain-wall behavior during reversal and thermally activated antiferromagnetic reversal in an exchange-biased NiO/NiFe bilayer" (2003). Faculty Publications: Materials Research Science and Engineering Center. 26.

https://digitalcommons.unl.edu/mrsecfacpubs/26

This Article is brought to you for free and open access by the Materials Research Science and Engineering Center at DigitalCommons@University of Nebraska - Lincoln. It has been accepted for inclusion in Faculty Publications: Materials Research Science and Engineering Center by an authorized administrator of DigitalCommons@University of Nebraska - Lincoln. 


\title{
Ferromagnetic domain-wall behavior during reversal and thermally activated antiferromagnetic reversal in an exchange-biased $\mathrm{NiO} / \mathrm{NiFe}$ bilayer
}

\author{
Z. Y. Liu and S. Adenwalla \\ Department of Physics and Astronomy, University of Nebraska-Lincoln, Lincoln, Nebraska 68588-0111
}

(Received 15 December 2002; published 30 May 2003)

\begin{abstract}
The magnetization reversal mechanism in an exchange-biased $\mathrm{NiO} / \mathrm{Ni}_{81} \mathrm{Fe}_{19}$ bilayer has been investigated using the magneto-optic Kerr effect and magnetic force microscopy imaging. The asymmetric reversal along the unidirectional axis and the two-step reversal process along the hard axis are promoted by ferromagnetic domain-wall behavior in the decreasing and increasing field branches of the as-deposited hysteresis loop, which is strongly related to the exchange coupling at the interface and the distribution of orientation of the net magnetization at the interface carried by the antiferromagnetic domains. The temperature dependence of the exchange bias and coercivity shows the thermally activated reversal of the net antiferromagnetic magnetization, which improves the unidirectional anisotropy at the interface or induces a new unidirectional axis, depending on whether the measuring field is along the unidirectional or hard axis of the as-deposited sample.

DOI: 10.1103/PhysRevB.67.184423

PACS number(s): 75.70.Ak, 75.70.Cn, 75.70.Kw, 75.30.Gw
\end{abstract}

\section{INTRODUCTION}

When a ferromagnetic (FM)-antiferromagnetic (AF) bilayer is grown or cooled in a magnetic field, the hysteresis loop of the FM layer is shifted from the origin by an amount known as the exchange bias $H_{E} \cdot{ }^{1,2}$ The macroscopic exchange bias effect has received extensive attention because of its technological applications and lack of fundamental understanding. Recent experimental and theoretical studies have shown that the existence of AF domains is a necessary condition for the appearance of exchange bias in FM-AF systems. Theoretical models have suggested both parallel and perpendicular domain walls in the AF layer. Mauri et al. ${ }^{3}$ suggest that a domain wall (DW) forms in the AF layer parallel to the interface while the magnetization of the FM layer rotates. Some experimental results have claimed the existence of parallel DW's. ${ }^{4,5}$ In models by Malozemoff ${ }^{6}$ and Nowak et al., ${ }^{7}$ the AF layer breaks up into domains with the DW's perpendicular to the interface due to interface roughness or volume defects when the sample is field cooled to below the Néel temperature, and the AF domains are frozen in by the AF anisotropy. The domain state (DS) model by Nowak et al. ${ }^{7}$ suggests that each AF domain carries a net magnetization that determines the exchange bias of the FM-AF systems. This model has been used to give a successful explanation of some experimental data. ${ }^{8}$ Owing to the unidirectional exchange coupling at the interface between the $\mathrm{FM}$ and $\mathrm{AF}$ bilayers, the existence of domains in the AF layer has strong effect on the DW formation and its behavior in the FM layer, ${ }^{9}$ which is strongly related to the asymmetric reversal process observed in the exchange-biased FM-AF bilayers. The difference in nucleation sites on either side of the loop of the FM layer has been observed experimentally to be the reason for the asymmetric shape of the loop. ${ }^{10,11}$ For some materials, polarized neutron reflectometry, ${ }^{12-14}$ Lorentz transmission electron microscopy, ${ }^{15,16}$ magnetometry, ${ }^{13,17}$ and magnetotransport measurements ${ }^{13}$ have observed that the reversal of the FM magnetization occurs via either DW motion or magnetization rotation on opposite sides of the same hysteresis loop. The observation of ferromagnetic DW nucleation and its behavior during the reversal process offers strong indirect information about the $\mathrm{AF}$ domain configuration in the AF layer, even though it is difficult to observe directly the AF domain structure.

The exchange bias is strongly affected by the thermal stability of the spin structure at the interface between the FM and AF bilayers. The interface spin structure does not remain stable below the Néel temperature if large enough fields are applied. ${ }^{18}$ It is well known that the reversal in the AF layer occurs due to the exchange coupling across the interface if the exchange-biased FM layer reverses. ${ }^{15,16,19}$ The AF reversal can happen either through the coherent rotation of singledomain particles ${ }^{20,21}$ or through the nucleation and growth of the AF domains. ${ }^{6,22,23}$ For both cases, the AF reversal has been driven by the thermal activation over an energy barrier distribution of some form. ${ }^{20,24}$ The thermally activated reversal in the AF layer makes the FM reversal more complicated through the exchange coupling across the interface, being responsible for many unique features observed for the FM-AF systems such as the asymmetric reversal, enhanced coercivity, and training effect. ${ }^{15,16,19}$

Above the Néel temperature $\left(T_{N}=523 \mathrm{~K}\right)$ the $\mathrm{NiO}$ lattice has a perfect fcc rocksalt structure. Below the Néel temperature, there is a small rhombohedral contraction of the crystal along different $\langle 111\rangle$ axes and the crystallographic twinning leads to so-called $T$ (twin) domains in which the spins lie in ferromagnetic $\{111\}$ planes with adjacent planes exhibiting antiferromagnetic alignment. Four principle $T$ domains correspond to the possible $\langle 111\rangle$ directions, each of which may further split into three different $S$ (spin) domains with the spins along three possible directions, e.g., [ $\overline{2} 11],[1 \overline{2} 1]$, and $[11 \overline{2}] .{ }^{25}$ Recent experimental results have shown that the domains in the $\mathrm{NiO}$ layer play an important role in the exchange bias of $\mathrm{FM}-\mathrm{NiO}$ systems. ${ }^{23,26-28}$

In this paper, we have used magneto-optic Kerr effect (MOKE) and magnetic force microscopy (MFM) imaging to investigate the ferromagnetic DW nucleation and its behavior during the magnetization reversal and the thermally in- 
duced variation of the unidirectional axis in an exchangebiased $\mathrm{NiO} / \mathrm{Ni}_{81} \mathrm{Fe}_{19}$ bilayer. The paper is arranged as follows. In the following section, sample preparation and detailed experimental measurements are described. The experimental results and discussion are offered in Sec. III. Finally, a summary is offered.

\section{SAMPLE PREPARATION AND EXPERIMENTAL MEASUREMENTS}

The $\mathrm{NiO}(45 \mathrm{~nm}) / \mathrm{NiFe}(15 \mathrm{~nm})$ bilayer was grown on a $\mathrm{Si}(100)$ substrate by $\mathrm{rf}$ and dc magnetron sputtering from $\mathrm{NiO}$ and NiFe targets. For prevention of oxidation, a 10-nm $\mathrm{Cu}$ layer was coated on top of the $\mathrm{NiFe}$ layer. The Ar pressure was 3 mTorr and the base pressure was $4 \times 10^{-7}$ Torr. No external field was applied during sample preparation, but there was a stray field of $\sim 8$ Oe from the gun in the plane of the substrate. X-ray diffraction shows the polycrystalline structure of $\mathrm{NiO}$ with a mixture of (111) and (200) orientations and the highly (111) textured NiFe.

The magnetization components parallel $\left(M_{l}\right)$ and perpendicular $\left(M_{t}\right)$ to the magnetic field were determined by MOKE magnetometry. A $660-\mathrm{nm}$ laser with $s$ polarization was used as light source. The magnetic field was applied in the plane of the sample and parallel to the incident plane of light. First, the longitudinal $M_{l}-H$ loop was obtained, after which the magnet together with the sample was turned $90^{\circ}$ with no change in the other parts and the transverse $M_{t}-H$ loop was measured (for details about the measurement of $M_{t}-H$ loop, see Ref. 29).

MFM images were obtained using low magnetic stray field and high coercivity MFM tips, with magnetization perpendicular to the sample surface. Thus, the MFM images highlight the out-of-plane magnetization component of the sample. The light and dark contrast corresponds to the strength of the stray field gradient on the sample surface. The as-deposited sample was initially saturated in the 450-Oe field. With the field being stepped down to -450 Oe, MFM images at different fields were obtained in the decreasing field branch. Then the field was increased to 450 Oe and MFM images at different fields were taken in the increasing field branch. Images in the same hysteresis loop were obtained from the same scanning area of $20 \mu \mathrm{m} \times 20 \mu \mathrm{m}$ without lifting the tip.

Two pieces of as-deposited sample were heated up to $250^{\circ} \mathrm{C}$. During the heating, a series of longitudinal $M_{l}-H$ loops at different temperatures was measured with the field applied parallel and perpendicular to the unidirectional axis (UA). After each measurement of the $M_{l}-H$ loop, the field was set to zero and the sample was heated to the next higher temperature in the remanent state. After being kept at $250{ }^{\circ} \mathrm{C}$ for $10 \mathrm{~min}$, the two pieces of sample were cooled down to room temperature in an applied field of $2 \mathrm{kOe}$ parallel and perpendicular to the UA, respectively. After cooling, the $M_{l^{-}} H$ loops parallel and perpendicular to the direction of the cooling field were measured.

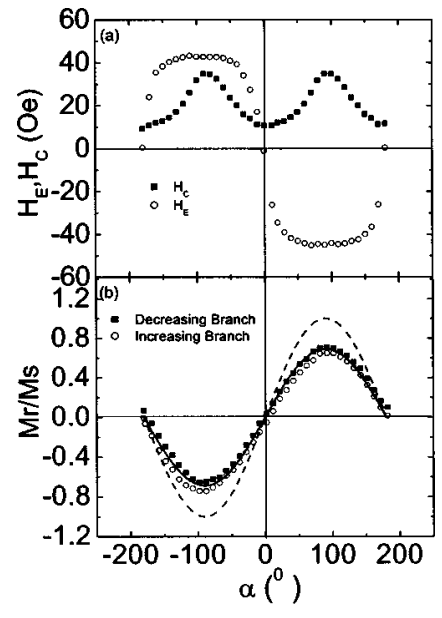

FIG. 1. The angular dependences of (a) the exchange bias and coercivity and (b) the remanent magnetization for the as-deposited $\mathrm{NiO} / \mathrm{NiFe}$ bilayer. $\alpha$ is the angle between the applied field and the hard axis. In (b), the solid line is the fit according to $C \sin \alpha$ with $C=0.69$ and the dashed line is given by $\sin \alpha$ from the rotation model.

\section{RESULTS AND DISCUSSION}

\section{A. Angular dependence of exchange bias, coercivity, and remanence}

It is easy to find the hard axis, along which the hysteresis loop is not shifted. By finding the hard axis and then varying the in-plane angle $\alpha$ of the applied field with respect to the hard axis, a series of $M_{l}-H$ loops was measured for the asdeposited sample. The coercive fields $H_{C D}$ and $H_{C I}$ for the decreasing and increasing field branches, respectively, are deduced, and then the coercive field $H_{C}=\left[H_{C I}-H_{C D}\right] / 2$ and the exchange bias $H_{E}=\left[H_{C I}+H_{C D}\right] / 2$ are obtained. The remanent magnetizations $M_{r}$ for both the decreasing and increasing field branches are also derived. The angular dependences of $H_{E}, H_{C}$, and $M_{r}$ are shown in Figs. 1(a) and 1(b). Both the exchange bias and the remanence display unidirectional symmetry, while the coercivity $H_{C}$ shows uniaxial symmetry of the NiFe layer.

Figure 2 displays the $M_{l}-H$ and $M_{t}-H$ loops when the applied field is parallel and perpendicular to the UA. With the field along the UA, the $M_{l^{-}} H$ loop [Fig. 2(I)] shows a large exchange bias of $H_{E}=-45 \mathrm{Oe}$ and the $M_{t}-H$ loop [Fig. 2(II)] shows a strongly asymmetric shape; the sign of $M_{t}$ does not vary with the field. When the field is perpendicular to the UA, the $M_{l}-H$ loop [Fig. 2(III)] is composed of two separate half loops asymmetrically shifted in opposite directions, suggesting that a two-step reversal process occurs along the hard axis (HA). The $M_{t}-H$ loop along the HA also shows that the sign of $M_{t}$ does not vary with the field.

To understand the phenomenon that the sign of $M_{t}$ does not vary with the field, let us consider the Stoner-Wohlfarth mode $^{30}$ and its extension to the exchange-biased FM-AF bilayers by Xi, Kryder, and White. ${ }^{31}$ By defining the coercivity $H_{C 0}=2 K_{\mathrm{FM}} / M_{S}$ and $H_{E 0}=2 \sqrt{A_{\mathrm{AF}} K_{\mathrm{AF}}} / M_{S} t_{\mathrm{FM}}$ (where $K_{\mathrm{FM}}$ and $K_{\mathrm{AF}}$ are the $\mathrm{FM}$ uniaxial anisotropy and the $\mathrm{AF}$ anisotropy, respectively, $A_{\mathrm{AF}}$ is the exchange constant of the 


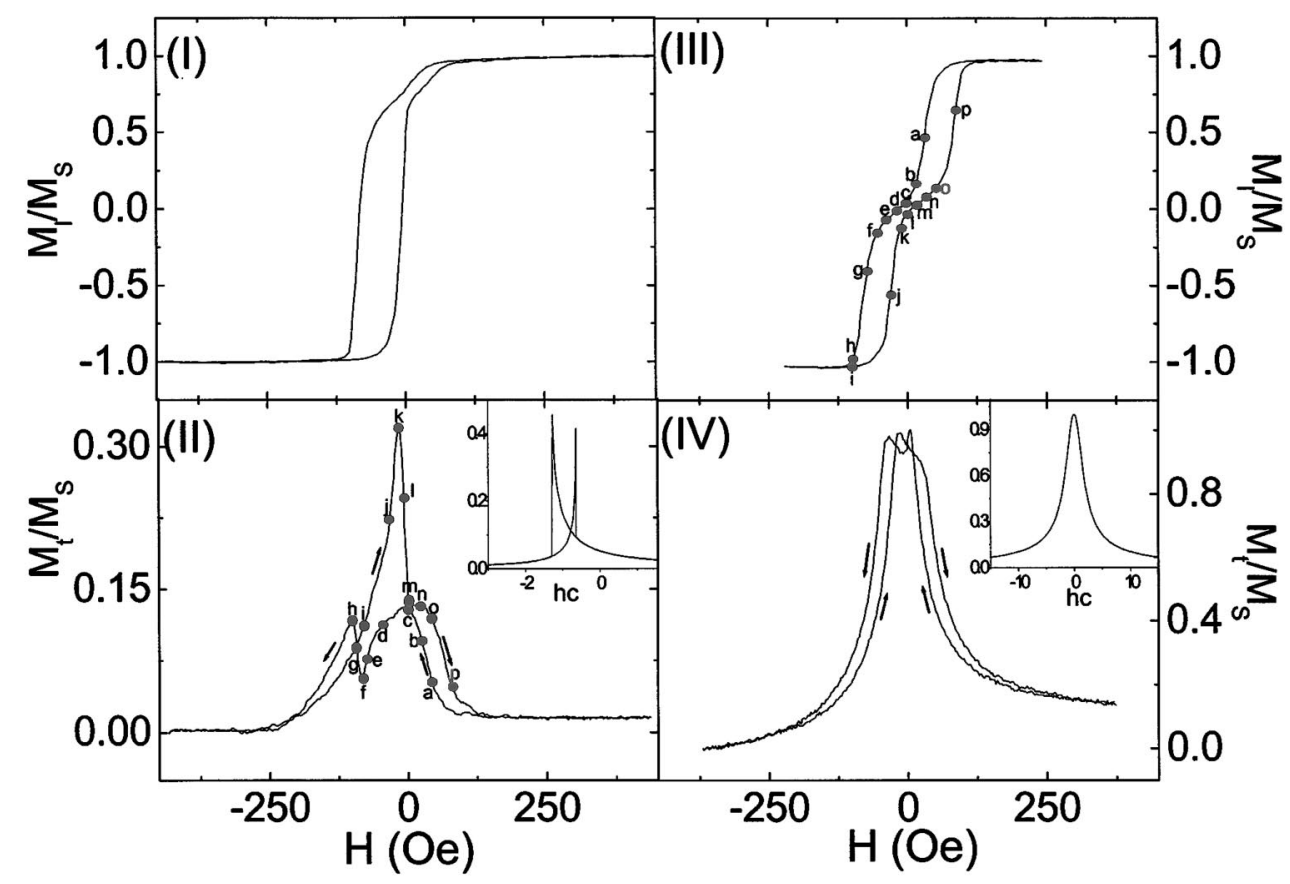

FIG. 2. The $M_{l}-H$ and $M_{t}-H$ loops along the as-deposited unidirectional (I and II) and hard (III and IV) axes. The insets in (II) and (IV) are the calculated $M_{t}-H$ loops at $\alpha=87^{\circ}$ and $0^{\circ}$, respectively, from the rotation model. In the calculation, the scaled coercivity $h_{c}$ is taken to be 0.5 .

AF layer, $M_{S}$ and $t_{\mathrm{FM}}$ are the FM saturation magnetization and thickness, respectively), the magnetic energy per unit area of the exchange-biased FM-AF bilayer scaled by $H_{E 0}$ can be expressed as ${ }^{31}$

$$
\epsilon=0.5 h_{c} \sin \theta^{2}-\cos \theta-h \cos (\alpha+\theta),
$$

where $h_{c}=H_{C 0} / H_{E 0}, h=H / H_{E 0}, \alpha$ is the angle between the applied field and the HA, and $\theta$ is the angle between the magnetization and the UA.

The value of $h_{c}$ indicates the strength of the FM uniaxial anisotropy relative to the exchange-coupling strength at the interface. By choosing a value of $h_{c}$ and finding the angle $\theta_{0}$ at which the energy is a minimum, the $M_{l}-H$ and $M_{t}-H$ loops can be determined by $M_{S} \cos \left(\alpha+\theta_{0}\right)$ and $M_{S} \sin (\alpha$ $+\theta_{0}$ ), respectively. Our detailed calculation ${ }^{32}$ has shown that the magnetization reversal depends strongly on the competition between the exchange coupling at the interface and the uniaxial anisotropy of the FM layer. If the exchange coupling is stronger than the uniaxial anisotropy, i.e., $h_{c}<1$, the sign of $M_{t}$ will not vary with the field, suggesting that the magnetization rotates on the same side of the field orientation in both the decreasing and increasing branches. The insets in Figs. 2(II) and 2(IV) give two calculated $M_{t}-H$ loops at the angles of $\alpha=87^{\circ}$ and $0^{\circ}$ with $h_{c}=0.5$, respectively, showing clearly that the sign of $M_{t}$ does not vary with the field if $h_{c}<1$. Under the condition of $h_{c}<1$, the calculated remanence $M_{r}$ is determined by $\sin \alpha$, displaying unidirectional symmetry. In Fig. 1(b), the experimental angular dependence of remanence $M_{r}$ is fitted according to $C \sin \alpha$ with $C$ $=0.69$ less than 1 expected from the rotation model. The fitting displays unidirectional symmetry of the remanence
$M_{r}$. Even though the rotation model can explain some general features of the hysteresis loop such as the unchanging sign of $M_{t}$ and the unidirectional symmetry of the remanence $M_{r}$, it cannot explain the details displayed in the hysteresis loop such as the strong asymmetric shape of the $M_{t}-H$ loop parallel to the UA and the two-step reversal process shown by the $M_{l}-H$ loop along the HA. These detailed features must be related to DW nucleation and behavior during the reversal. To better understand the DW nucleation and its behavior, MFM images at different fields in the hysteresis loops have been obtained and shown in the following section.

\section{B. MFM images}

\section{Along the unidirectional axis}

The $M_{t}-H$ loop parallel to the UA [Fig. 2(II)] demonstrates a strong asymmetric magnetization reversal in the decreasing and increasing field branches. MFM images at different fields in the decreasing and increasing field branches show that the asymmetric reversal mechanism is strongly related to the asymmetry of the DW nucleation and its behavior. Figures 3 and 4 give the MFM images at different fields in the decreasing and increasing field branches, respectively.

In the decreasing field branch, images (a) to (e) in Fig. 3 show that the contrast of the DW becomes sharper and sharper with decreasing field, but the domain profile does not change, suggesting that the DW's grow slowly and are completely pinned. Around the coercive field, image (f) shows that the DW's are unpinned. The unpinned DW's disappear quickly, leaving the FM layer in a single domain as shown in images (g) and (h). 

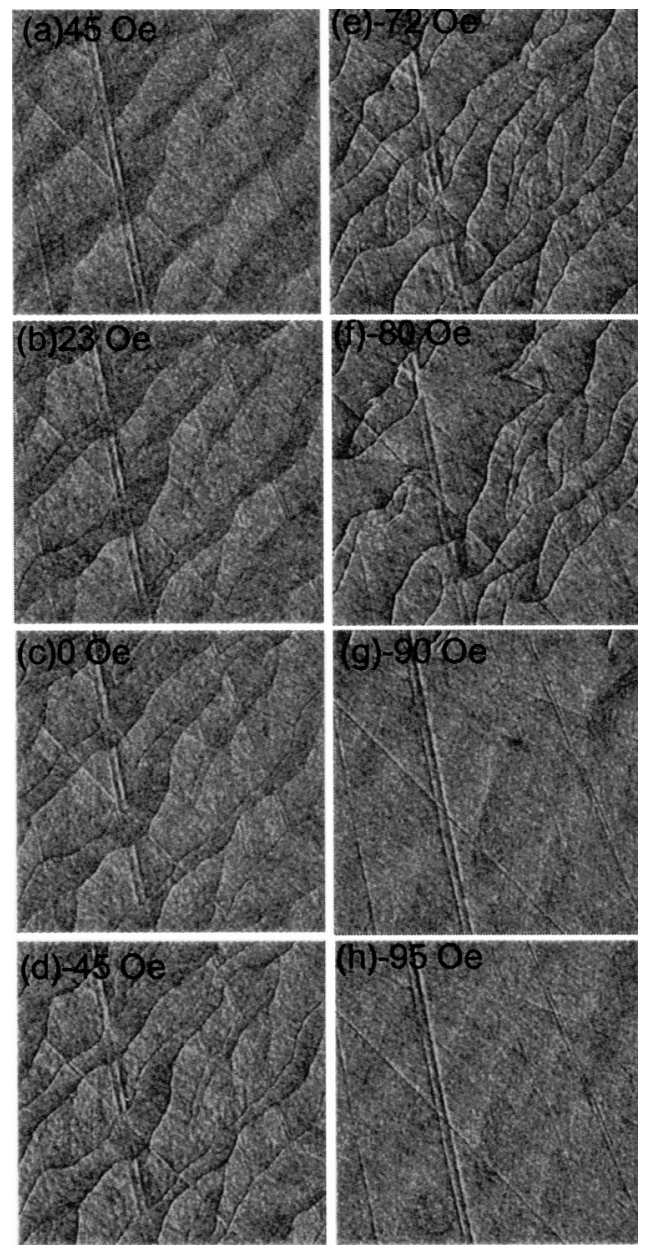

FIG. 3. The MFM images at different fields in the decreasing field branch of the hysteresis loop along the as-deposited unidirectional axis. The panels (a)-(h) correspond to those in Fig. 2(II).

This DW behavior is caused by the competition between the Zeeman energy and the exchange coupling at the interface. As the field decreases away from the positive saturation field, it will drag the FM moments away from the UA, but the exchange coupling at the interface will block this rotation. According to the DS model by Nowak et al., ${ }^{7}$ the AF layer is composed of domains, each of which carries a net $\mathrm{AF}$ magnetization at the interface. In $\mathrm{NiO}$, there exist 24 possible domains in total with different easy axes. Owing to the local random interface and the nonuniform stray field from the sputtering gun during sample preparation, there will be a distribution of orientations of the net AF magnetization at the interface. Thus, blocking the FM moment rotation by the exchange coupling at the interface is locally nonuniform, leading to the FM DW nucleation from the interface with the DW's pinned by the exchange coupling. As the field decreases to the coercive field [-80 Oe at in Fig. 3(f)], the Zeeman energy dominates, overcoming the exchange coupling to cause the unpinning of the DW's. The unpinned DW's disappear quickly to induce the sharp jump around the coercive field observed in the $M_{t}-H$ loop in Fig. 2(II).

In the increasing field branch, images in Fig. 4 show quite different DW nucleation and behavior. When the field in-
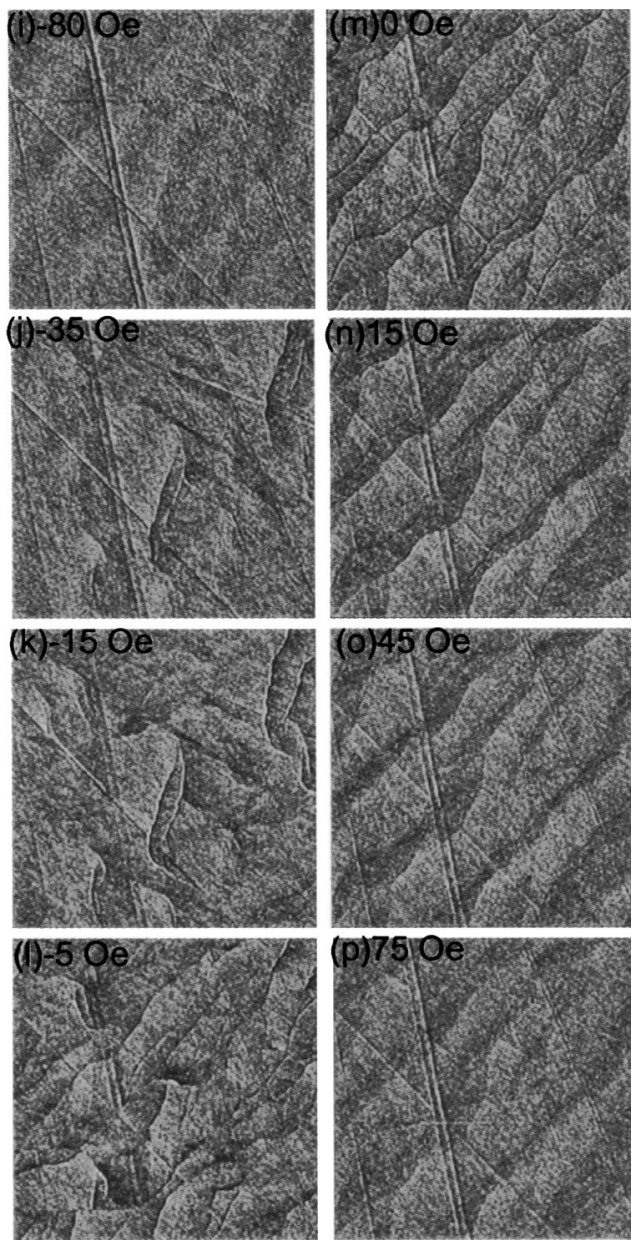

FIG. 4. The MFM images at different fields in the increasing field branch of the hysteresis loop along the as-deposited unidirectional axis. The panels (i)-(p) correspond to those in Fig. 2(II). The straight lines in the images of Fig. 3 and 4 are due to the scratches from cleaning the sample.

creases away from the negative saturation field, the sample is basically in a single domain as shown in images (i) to (j) except for a few DW's due to the defects in the FM layer. The reversal is towards the UA. Thus, the FM moments rotate easily towards this direction with the field to minimize the exchange-coupling energy. With increasing field close to zero, the old DW's due to defects in the FM layer disappear quickly, and simultaneously some new DW's are nucleated as displayed in images (i) and (m). The quick disappearance of old DW's and simultaneous appearance of new DW's give rise to the sharp drop close to zero field observed in the $M_{t}-H$ loop in Fig. 2(II). Comparing images (c) and (m) shows that the domain profiles are the same at the remanent states in both the decreasing and increasing branches. As the field increases away from zero, images (n) to (p) show that the DW's are pinned in position but the contrast of the DW's becomes less and less sharp until they disappear. The exchange-coupling energy dominates close to zero field. Due to the distribution of orientations of the net AF magnetization, the local exchange coupling cannot uniformly block the FM moment rotation caused by the field. That will again lead to the quick nucleation of the DW's in the FM layer, display- 

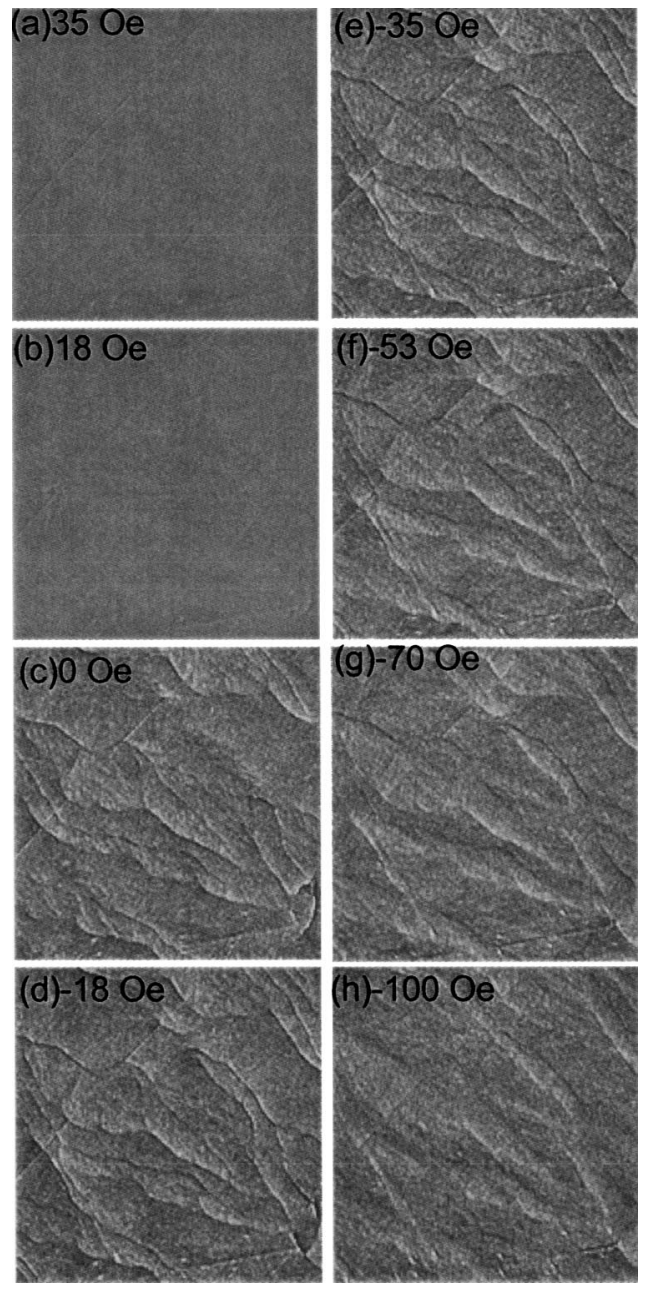

FIG. 5. The MFM images at different fields in the decreasing field branch of the hysteresis loop along the as-deposited hard axis. The panels (a)-(h) correspond to those in Fig. 2(III).

ing a domain configuration identical to that in the decreasing field branch. With the DW's pinned by the exchange coupling at the interface, the FM moments inside the domains are rotated towards the UA with increasing field toward the positive saturation field, leading to the gradual untwisting of the DW's until their disappearance.

The MFM images reveal clearly that the asymmetric shape of the $M_{t}-H$ along the UA is caused by a different reversal mechanism in the decreasing and increasing field branches. Similar asymmetry has also been observed in several other experiments. ${ }^{12-17}$ The exchange coupling at the interface between FM and AF bilayers is believed to play an important role in the asymmetric reversal mechanism.

\section{Along the hard axis}

The $M_{l}-H$ loop in Fig. 2(III) reveals a two-step magnetization reversal process along the HA, which is related to DW nucleation as revealed by the MFM images in the decreasing and increasing field branches in Figs. 5 and 6.

In the decreasing field branch (see Fig. 5), as the field was stepped down from 450 Oe to zero, images (a) and (b) show that no DW's exist, suggesting that the sample is in a single
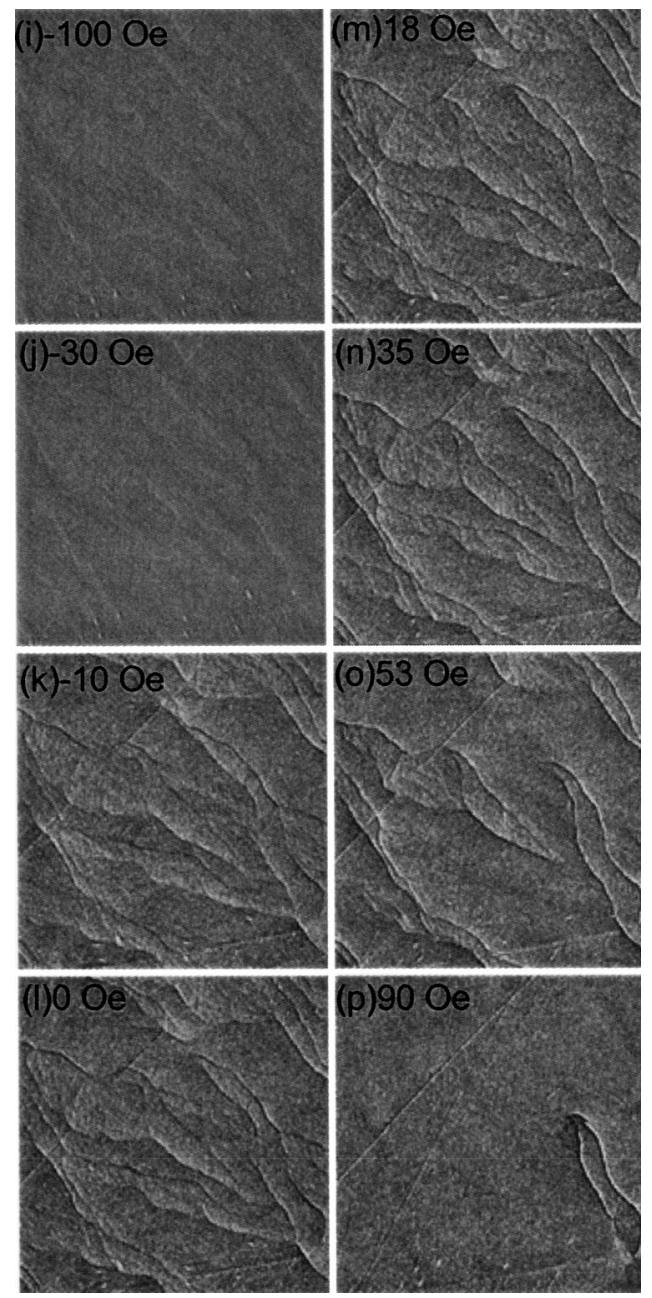

FIG. 6. The MFM images at different fields in the increasing field branch of the hysteresis loop along the as-deposited hard axis. The panels (i) $-(\mathrm{p})$ correspond to those in Fig. 2(III).

domain and the FM moments rotate to the UA with the field. When the field decreases to zero, DW's appear as shown in image (c). Further decreasing the field from zero, images (c)-(h) reveal that the domain configuration does not change, i.e., the DW's are pinned. Only the FM moments inside domains rotate. With further decreasing field towards a large negative field, the contrast of DW's become less and less sharp until they disappear.

This different reversal mechanism in a different range of fields is determined by the competition between the exchange-coupling energy at the interface and the Zeeman energy. As the field decreases towards zero, the reversal is towards the UA and the FM moments can easily rotate back to this direction. Around zero field, the energy is favorable for the exchange-coupling energy at the interface. Thus, with further decreasing field away from zero, the exchange coupling generally blocks the rotation of the FM moments, but the blocking is not uniform due to the local distribution of orientations of the net AF magnetization, leading to the quick DW nucleation with the DW's pinned in position. Only the FM moments inside domains are rotated. With further decreasing field towards the negative large field, the rotation of 
FM moments inside domains causes the gradual untwisting of the DW's until their disappearance.

In the increasing field branch (see Fig. 6), as the field increases away from the negative saturation field, the reversal is towards the UA, and it is easy for the FM moments to rotate back to this direction to minimize the exchangecoupling energy. Thus, the images (i) and (j) reveal that the FM layer is in a single domain. With the increasing field close to zero, the exchange-coupling energy overcomes the Zeeman energy, leading to the quick DW nucleation in the FM layer as shown by images (k) and (1). With further increasing field away from zero, images $(\mathrm{k})-(\mathrm{n})$ show that the domain pattern does not change, but the contrast of the DW's becomes sharper, implying that the DW's are pinned by the exchange coupling at the interface and grow slowly. With increasing field towards large field, the Zeeman energy overcomes the exchange coupling, leading to the unpinning of the DW's. Images (o) and ( $\mathrm{p}$ ) have shown that the unpinned DW's shrink quickly, leaving the FM layer in a single domain at large field.

The MFM images have shown clearly that the two-step magnetization reversal process along the HA is strongly related to the difference in the reversal mechanism in different range of field. In both the decreasing and increasing field branches, as the field decreases from (increases towards) zero from the saturation field, the reversal is towards the UA, and it is easy for the FM moments to rotate back to this direction to minimize the exchange-coupling energy. As the field is around zero, the exchange-coupling energy becomes dominant. Driven by the exchange coupling at the interface, the FM DW nucleation occurs quickly due to the existence of $\mathrm{AF}$ domains and the DW's are pinned in position by the exchange coupling. As the field decreases (increases) away from zero, only the FM moments inside domains rotate.

A careful check shows that the domains in both the decreasing and increasing field branches are basically in the same pattern. This strongly suggests that the ferromagnetic DW nucleation is related to the AF domain configuration through the exchange coupling at the interface.

\section{Temperature dependence of exchange bias and coercivity}

\section{Along the unidirectional axis}

Figure 7 gives the $M_{l}-H$ loops at some typical different temperatures. Above $110^{\circ} \mathrm{C}$, the shape of the $M_{l}-H$ loop becomes quite square. The temperature dependence of the exchange bias $H_{E}$ and coercivity $H_{C}$ obtained from the $M_{l^{-}} H$ loops are shown in Figs. 8(a) and 8(b). Figure 8(a) displays the monotonic decrease of the coercivity $H_{C}$ with increasing temperature. However, Fig. 8(b) shows a two-step process in the monotonic decrease of $H_{E}$. Below $110^{\circ} \mathrm{C}, H_{E}$ drops quickly with temperature, but above $110^{\circ} \mathrm{C}, H_{E}$ drops much more slowly and disappears at the blocking temperature of $T_{B}=180^{\circ} \mathrm{C}$.

\section{Along the hard axis}

Figure 9 shows the $M_{l}-H$ loops at some typical temperatures with the field parallel to the as-deposited HA. With

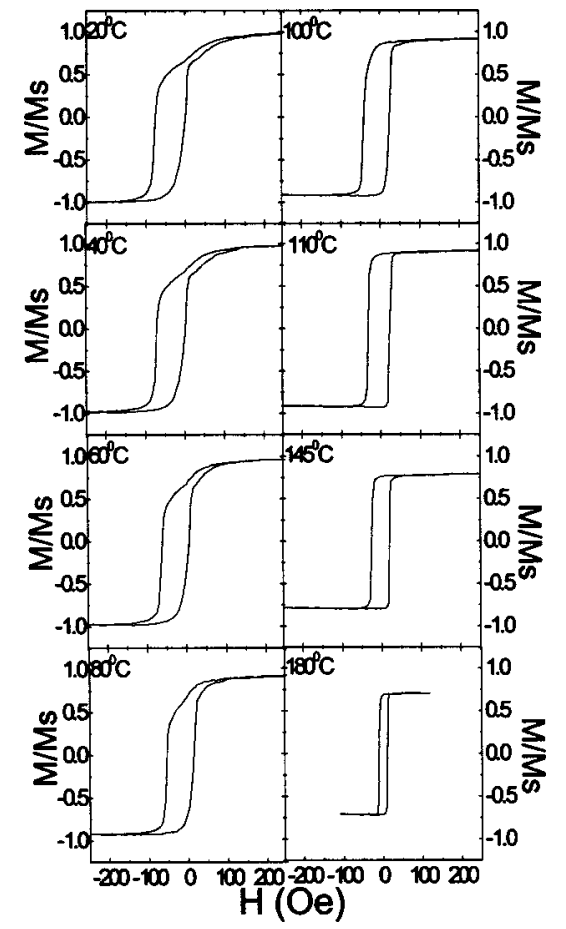

FIG. 7. The $M_{l}-H$ loops at different temperatures during heating with the measuring field along the as-deposited unidirectional axis. All loops are normalized by the saturation magnetization at room temperature.

increasing temperature, the two-step reversal process becomes weaker and disappears completely when the temperature reaches $110^{\circ} \mathrm{C}$, above which the $M_{l^{-}} H$ loop becomes square. However, the loop shift is not zero as expected and decreases monotonically with increasing temperature above $110^{\circ} \mathrm{C}$

In order to understand the temperature dependence of the loop shift along the hard axis, we define the exchange bias and coercivity for the upper and lower loops in Fig. 8(c), and their temperature dependences are shown in Figs. 8(a) and 8(b). Below $110^{\circ} \mathrm{C}$, the coercive fields $H_{U C}$ and $H_{L C}$ for both the upper and lower loops, respectively, are almost the same and independent of the temperature, but they increase slowly with increasing temperature above $110^{\circ} \mathrm{C}$ and reach a maximum at $140^{\circ} \mathrm{C}$, after which they decrease monotonically. Below $140^{\circ} \mathrm{C}$, they are much lower than that along the UA but become comparable above $140{ }^{\circ} \mathrm{C}$. The temperature dependences of the exchange bias $H_{U E}$ and $H_{L E}$ for the upper and lower loops are almost symmetric, displaying a twostep process identical to that along the UA. Below $110^{\circ} \mathrm{C}$, the exchange bias for both loops drop quickly with temperature, but above $110^{\circ} \mathrm{C}$, they decrease very more slowly and disappear at the same blocking temperature of $T_{B}=180^{\circ} \mathrm{C}$ as that along the UA. Figure 8(d) gives the temperature dependence of the remanent magnetization in the decreasing field branch. With increasing temperature, the remanent magnetization increases sharply and reaches a maximum at $100^{\circ} \mathrm{C}$, after which the remanent magnetization becomes the same as the saturation magnetization.

For the as-deposited sample, there exists a distribution of orientations of the net $\mathrm{AF}$ magnetization at the interface, 


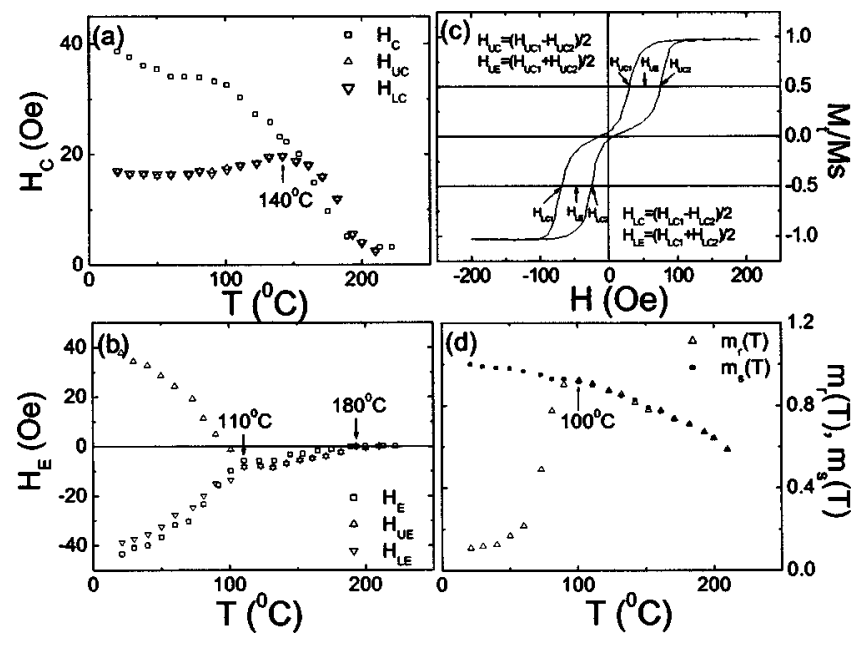

FIG. 8. (a) The temperature dependences of the coercivity with the measuring field along the as-deposited unidirectional $\left(H_{C}\right)$ and hard $\left(H_{U C}, H_{L C}\right)$ axes. (b) The temperature dependences of the exchange bias with the measuring field along the as-deposited unidirectional $\left(H_{E}\right)$ and hard $\left(H_{U E}, H_{L E}\right)$ axes. (c) The $M_{l}-H$ loop along the as-deposited hard axis at room temperature, in which the exchange fields of $H_{U E}$ and $H_{L E}$ and the coercivities of $H_{U C}$ and $H_{L C}$ for the upper and lower loops, respectively, are defined. (d) The temperature dependences of $m_{s}(T)$ and $m_{r}(T)$ in the decreasing field branch of the $M_{l}-H$ loop along the as-deposited hard axis. $m_{s}(T)$ and $m_{r}(T)$ are the normalized saturation and remanent magnetizations by the saturation magnetization at room temperature, respectively.

which is frozen in by the AF anisotropy. With increasing temperature, the AF anisotropy drops sharply, dropping by $\sim 60 \%$ at $100{ }^{\circ} \mathrm{C} .{ }^{33}$ At high temperature, the AF anisotropy will become too weak to keep the net AF magnetization fixed. Driven by the thermal activation, the remanent magnetization can drag the net AF magnetization towards the field direction through the exchange coupling.

When the $M_{l}-H$ loop is measured with the field applied along the UA during heating, the FM remanent magnetization lies along the UA. When the temperature is higher than $110^{\circ} \mathrm{C}$, the thermal activation becomes strong enough to overcome the AF anisotropy. The FM remanent magnetization drags the AF net magnetization to the UA, narrowing the distribution of the net AF magnetization direction around the UA and then improving the unidirectional anisotropy. This improvement causes the $M_{l}-H$ loop to become square when the temperature is higher than $110^{\circ} \mathrm{C}$.

For the measurement of the $M_{l}-H$ loop with the field applied along the HA during heating, the FM remanent magnetization lies along the HA and increases sharply with temperature, reaching the saturation magnetization at $100^{\circ} \mathrm{C}$ as shown in Fig. 8(d). When the temperature is higher than $100{ }^{\circ} \mathrm{C}$, the thermal activation is strong enough to overcome the AF anisotropy, and the FM remanent magnetization also becomes large enough so that it drags the net AF magnetization to flip to the field direction through the exchange coupling at the interface, inducing a new unidirectional axis along the field direction. Afterwards, when the sample is heated to higher temperature, the two-step magnetization re-

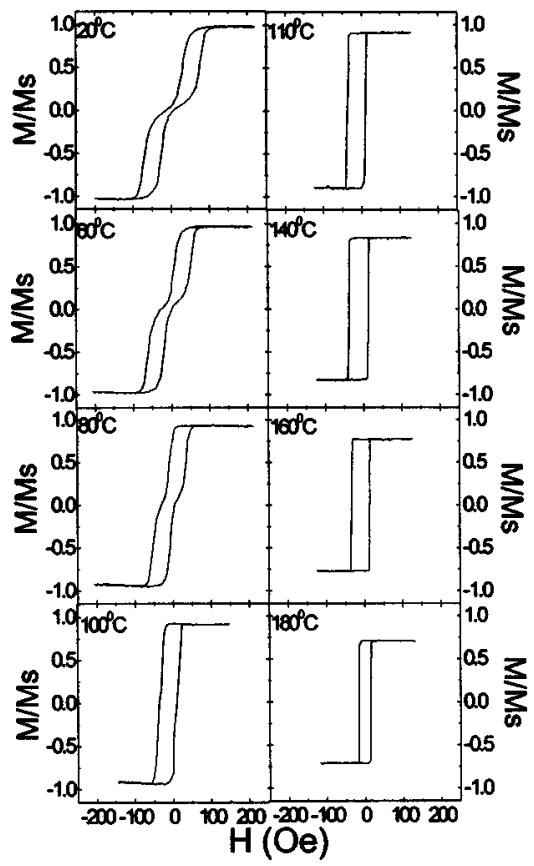

FIG. 9. The $M_{l}-H$ loops at different temperatures during heating with the measuring field along the as-deposited hard axis. All loops are normalized by the saturation magnetization at room temperature.

versal process is erased completely and the $M_{l^{-}} H$ loop becomes square, displaying almost the same loop shift and coercivity as the $M_{l}-H$ loop along the UA.

Figures 10(a) and 10(b) display the $M_{l}-H$ loops parallel and perpendicular to the cooling field after field cooling. Comparing Fig. 10(a) to 10(b) shows clearly that regardless of whether the cooling field is parallel or perpendicular to the as-deposited UA, the $M_{l}-H$ loops along the direction of the cooling field are in a square shape, displaying the almost identical loop shift of $\sim 38$ Oe, a little less than 45 Oe for the as-deposited sample. The $M_{l}-H$ loops perpendicular to the

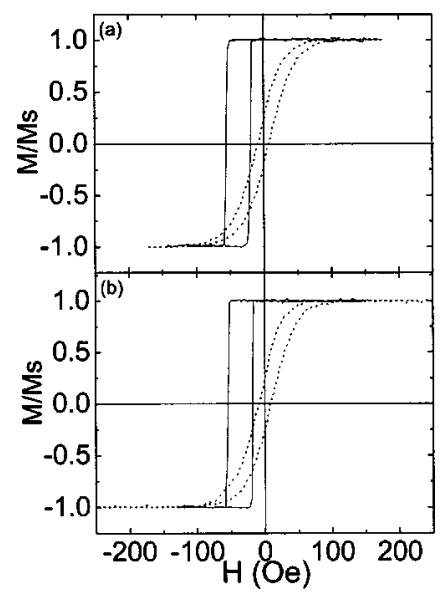

FIG. 10. The $M_{l^{-}} H$ loops at room temperature after field cooling along the as-deposited (a) unidirectional and (b) hard axes, respectively. Solid and dashed lines represent the loops measured with the field applied parallel and perpendicular to the direction of the cooling field, respectively. 
cooling field show the feature of hysteresis loop along the hard axis. It suggests that the unidirectional anisotropy is improved or a new unidirectional axis is induced parallel to the cooling field, depending on whether the cooling field is parallel to the as-deposited UA or HA.

\section{SUMMARY}

The angular dependence of the exchange bias and coercivity shows the development of an interfacial unidirectional anisotropy and a uniaxial anisotropy in the as-deposited $\mathrm{NiO} /$ $\mathrm{NiFe}$ bilayer. With the exchange coupling at the interface stronger than the uniaxial anisotropy, the angular dependence of the remanent magnetization of the FM layer also shows a unidirectional symmetry, and the magnetization component perpendicular to the field keeps the same sign during the reversal. The asymmetric shape of the $M_{l}-H$ loop along the as-deposited UA is promoted by the different reversal mechanism on opposite sides of the loop: In the decreasing field branch, the reversal is caused by FM DW nucleation and its behavior, but the reversal is basically through magnetization rotation in the increasing field branch. The twostep reversal process displayed by the $M_{l}-H$ loop along the as-deposited hard axis is promoted by the different reversal mechanism in different ranges of the field: in both the decreasing and increasing field branches, when the field decreases from (increases towards) the saturation field towards zero field, the reversal is towards the unidirectional axis and it is easy for the magnetization to rotate back to this direction to minimize the exchange coupling energy. However, when the field decreases (increases) away from zero field, it is energetically favorable for the exchange coupling, which will impede the FM moment rotation away from the as-deposited UA. Due to the distribution of the orientation of the net AF magnetization carried by the AF domains, the blocking is not uniform, leading to the quick FM DW nucleation with the DW's pinned by the exchange coupling at the interface. With further decreasing (increasing) the field towards the saturation field, the FM moments inside the domain are rotated to the field direction. On heating the as-deposited sample, when the temperature becomes higher than $100^{\circ} \mathrm{C}$, the thermal activation overcomes the AF anisotropy and the remanent magnetization drags the net AF magnetization towards the field direction. Depending on whether the measuring field is applied parallel or perpendicular to the as-deposited UA, the unidirectional anisotropy is improved or a new unidirectional axis is induced along the field direction and the hard axis is perpendicular to it.

\section{ACKNOWLEDGMENTS}

One of the authors (Z.Y.L.) would like to thank L. Gao for help with MFM imaging and thank Professor M.L. Yan for helpful discussions. This work was supported by NSF Grant No. DMR-9806308 and NSF MRSEC DMR-0213808.
${ }^{1}$ W. H. Meiklejohn and C. P. Bean, Phys. Rev. 102, 1413 (1956).

${ }^{2}$ J. Nogues and I. K. Schuller, J. Magn. Magn. Mater. 192, 204 (1999).

${ }^{3}$ D. Mauri, H. C. Siegmann, P. S. Bagus, and E. Kay, J. Appl. Phys. 62, 3047 (1987).

${ }^{4}$ P. Steadman, M. Ali, A. T. Hindmarch, C. H. Marrows, B. J. Hickey, S. Langridge, R. M. Dalgliesh, and S. Foster, Phys. Rev. Lett. 89, 077201 (2002).

${ }^{5}$ F. Y. Yang and C. L. Chien, Phys. Rev. Lett. 85, 2597 (2000).

${ }^{6}$ A. P. Malozemoff, Phys. Rev. B 35, 3679 (1987); J. Appl. Phys. 63, 3874 (1988).

${ }^{7}$ U. Nowak, K. D. Usadel, J. Keller, P. Miltenyi, B. Beschoten, and G. Guntherodt, Phys. Rev. B 66, 014430 (2002).

${ }^{8}$ J. Keller, P. Miltenyi, B. Beschoten, G. Guntherodt, U. Nowak, and K. D. Usadel, Phys. Rev. B 66, 014431 (2002).

${ }^{9}$ H. Matsuyama, C. Haginoya, and K. Koike, Phys. Rev. Lett. 85, 646 (2000).

${ }^{10}$ V. I. Nikitenko, V. S. Gomakov, L. M. Dedukh, Y. P. Kabanov, A. F. Khapikov, A. J. Shapiro, R. D. Shull, A. Chaiken, and R. P. Michel, Phys. Rev. B 57, R8111 (1998).

${ }^{11}$ V. I. Nikitenko, V. S. Gornakov, A. J. Shapiro, R. D. Shull, Kai Liu, S. M. Zhou, and C. L. Chien, Phys. Rev. Lett. 84, 765 (2000).

${ }^{12}$ M. R. Fitzsimmons, P. Yashar, C. Leighton, I. K. Schuller, J. Nohues, C. F. Majkrzak, and J. A. Dura, Phys. Rev. Lett. 84, 3986 (2000).

${ }^{13}$ C. Leighton, M. R. Fitzsimmons, P. Yashar, A. Hoffmann, J.
Nogues, J. A. Dura, C. F. Majkrzak, and I. K. Schuller, Phys. Rev. Lett. 86, 4394 (2001).

${ }^{14}$ M. Gierlings, M. J. Prandolini, H. Fritzsche, M. Gruyters, and D. Riegel, Phys. Rev. B 65, 092407 (2002).

${ }^{15}$ X. Portier, A. K. Petford-Long, A. de Morais, N. W. Owen, H. Laidler, and K. O'Grady, J. Appl. Phys. 87, 6412 (2000).

${ }^{16}$ Y. G. Wang, A. K. Petford-Long, T. Hughes, H. Laidler, K. O’Grady, and M. T. Kief, J. Magn. Magn. Mater. 242-245, 1073 (2002).

${ }^{17}$ C. Leighton and I. K. Schuller, Phys. Rev. B 63, 174419 (2001).

${ }^{18}$ J. Nogues, L. Morellon, C. Leighton, M. R. Ibarra, and I. K. Schuller, Phys. Rev. B 61, R6455 (2000).

${ }^{19}$ Y. G. Wang and A. K. Petford-Long, J. Appl. Phys. 92, 6699 (2002).

${ }^{20}$ E. Fulcomer and S. H. Charap, J. Appl. Phys. 43, 4184 (1972).

${ }^{21}$ C. Hou, H. Fujiwara, K. Zhang, A. Tanaka, and Y. Shimizu, Phys. Rev. B 63, 024411 (2000).

${ }^{22}$ M. D. Stiles and R. D. Michael, Phys. Rev. B 60, 12950 (1999).

${ }^{23}$ W. Zhu, L. Seve, R. Sears, B. Sinkovic, and S. S. Parkin, Phys. Rev. Lett. 86, 5389 (2001).

${ }^{24}$ R. L. Stamps, Phys. Rev. B 61, 12174 (2000).

${ }^{25}$ S. Saito, M. Miura, and K. Kurosawa, J. Phys. C 13, 1513 (1980).

${ }^{26}$ Z. Y. Liu and S. Adenwalla, Appl. Phys. Lett. 83, 2106 (2003).

${ }^{27}$ K. Takano, Ph.D. thesis, Physics Department, University of Calofornia at San Diego (1998).

${ }^{28}$ H. Ohldag, A. Scholl, F. Nolting, S. Anders, F. U. Hillebrecht, and J. Stohr, Phys. Rev. Lett. 86, 2878 (2001). 
${ }^{29}$ C. Daboo, J. A. C. Bland, R. J. Hicken, A. J. R. Ives, M. J. Baird, and M. J. Walker, Phys. Rev. B 47, 11852 (1993).

${ }^{30}$ E. C. Stoner and E. P. Wohlfarth, Philos. Trans. R. Soc. London 240, 599 (1948).
${ }^{31}$ H. Xi, M. H. Kryder, and R. M. White, Appl. Phys. Lett. 74, 2687 (1999).

${ }^{32}$ Z. Y. Liu and S. Adenwalla, J. Appl. Phys. 93, 3422 (2003).

${ }^{33}$ K. Kurosawa, M. Miura, and S. Saito, J. Phys. C 13, 1521 (1980). 